\author{
Revista Brasileira de Educação do Campo \\ Brazilian Journal of Rural Education \\ ARTIGO/ARTICLE/ARTÍCULO \\ DOI: http://dx.doi.org/10.20873/uft.rbec.e11885
}

\title{
Comunidades y aprendizaje informal: Las narrativas rurales de dos cooperativistas
}

Rocío Belén Martín ${ }^{1}$, (iD Emiliano Foresto ${ }^{2}$, (D) Danilo Silvio Donolo ${ }^{3}$

${ }^{1}$ Centro de Conocimiento, Formación e Investigación en Estudios Sociales, Consejo Nacional de Investigaciones Científicas y Técnicas - CONICET. Departamento de Enseñanza de la Ciencia y la Tecnología, Facultad de Ciencias Exactas, Físicas y Naturales, Universidad Nacional de Córdoba (UNC). Avenida Vélez Sarsfield 299. Córdoba Capital, Argentina. ${ }^{2}$ Instituto de Biotecnología Ambiental y Salud (INBIAS), CONICET. Departamento de Biología Agrícola. Facultad de Agronomía y Veterinaria. Universidad Nacional de Río Cuarto (UNRC). ${ }^{3}$ Consejo Nacional de Investigaciones Científicas y Técnicas CONICET. Universidad Nacional de Río Cuarto (UNRC).

Autor para correspondência/Author for correspondence: rbmartin@unc.edu.ar

RESUMEN. En educación la posibilidad de narrar y utilizar relatos persigue objetivos profundos desde los saberes, identidades, valores y visiones del mundo, que se relacionan con lo que se espera que sean los seres humanos. Por ello, es importante que en la escuela se incorporen narrativas informales en la enseñanza de diferentes disciplinas. Desde un estudio de casos múltiples se busca comprender desde las narrativas las formas y sentidos que denotan los contextos rurales. Del estudio participaron dos personas que forman parte de dos cooperativas; una cooperativa artística permacultural y otra agrícola tambera, ubicadas en la provincia de Córdoba, Argentina. Se describen y analizan los relatos atendiendo a tres dimensiones: trayectorias de participación en territorio, educación para y con la comunidad y prácticas cooperativas y soberanas; estos aspectos permiten analizar las narrativas y avanzar hacia una interacción en diversas ecologías y contextos. Entre los principales resultados, se observa que las trayectorias de participación laboral dentro de cada cooperativa responden en conjunto a diversas vivencias educativas y participativas que se entrelazan $\mathrm{y}$ resignifican de múltiples formas; otorgando sentido $\mathrm{y}$ permitiendo desarrollar nuevos modos de mirar, sentir y situarse en el mundo. Para finalizar se reflexiona en torno a las narrativas rurales como formas y sentidos para apuntar a nuevos aprendizajes y a prácticas novedosas.

Palabras clave: narrativas rurales, aprendizaje informal, comunidad de práctica, cooperativas. 


\title{
Communities and informal learning: The rural narratives of two cooperativists
}

\begin{abstract}
In education, the possibility of narrating and using stories pursues deep objectives based on knowledge, identities, values and worldviews, which are related to what human beings are expected to be. Therefore, it is important that schools incorporate informal narratives in the teaching of different disciplines. From a multiple case study we seek to understand from the narratives the forms and meanings that denote rural contexts. Two people who are part of two cooperatives participated in the study; a permacultural artistic cooperative and a dairy farming cooperative, located in the province of Córdoba, Argentina. The narratives are described and analyzed according to three dimensions: trajectories of participation in the territory, education for and with the community, and cooperative and sovereign practices; these aspects allow us to analyze the narratives and move towards an interaction in diverse ecologies and contexts. Among the main results, it is observed that the trajectories of labor participation within each cooperative respond as a whole to diverse educational and participatory experiences that are intertwined and re-signified in multiple ways; giving meaning and allowing the development of new ways of looking, feeling and situating oneself in the world. Finally, we reflect on rural narratives as forms and meanings to point to new learning and innovative practices.
\end{abstract}

Keywords: rural narratives, informal learning, community of practice, cooperatives 


\section{Comunidades e aprendizagem informal: As narrativas rurais de dois membros cooperativistas}

RESUMO. Na educação, a possibilidade de narrar e usar histórias busca objetivos profundos a partir do conhecimento, identidades, valores e visões de mundo, que estão relacionados com o que se espera que os seres humanos sejam. Portanto, é importante que as escolas incorporem narrativas informais no ensino de diferentes disciplinas. A partir de um estudo de caso múltiplo, procuramos entender a partir das narrativas as formas e significados que denotam os contextos rurais. O estudo envolveu duas pessoas que fazem parte de duas cooperativas; uma cooperativa de arte permacultural e uma cooperativa de laticínios, localizadas na província de Córdoba, Argentina. As narrativas são descritas e analisadas de acordo com três dimensões: trajetórias de participação no território, educação para e com a comunidade, e práticas cooperativas e soberanas; estes aspectos nos permitem analisar as narrativas e avançar em direção a uma interação em diversas ecologias e contextos. Entre os principais resultados, observa-se que as trajetórias de participação laboral dentro de cada cooperativa respondem como um todo a diversas experiências educativas e participativas que se entrelaçam e se resignam de múltiplas formas; dando sentido e permitindo o desenvolvimento de novas formas de olhar, sentir e se situar no mundo. Finalmente, refletimos sobre as narrativas rurais como formas e significados para apontar novas aprendizagens e novas práticas.

Palavras-chave: narrativas rurais, aprendizagem informal, comunidade de prática, cooperativas. 


\section{Introducción}

La psicología rural como campo de conocimiento se orienta a co-construir una mirada y una práctica desde la psicología que resulte apropiada $\mathrm{y}$ útil para las poblaciones rurales y sus problemáticas (Landini, 2015); atendiendo al abordaje sobre formas y prácticas educativas en el contexto rural, que no solo consideren a la educación formal sino también reconozcan actividades y aprendizajes informales. La psicología rural, encuentra confluencia con marcos y enfoques de la psicología educativa y la psicología ambiental; ya que actualmente desde la psicología educacional se empieza a considerar al aprendizaje en su diversidad de contextos. En este sentido, Barron y Bell (2016) advierten sobre un alejamiento respecto a conceptualizaciones del aprendizaje vinculadas a contextos específicos, comenzando a comprender al aprendizaje como un proceso ensamblado en diversos entornos, desdibujándose los límites entre lo formal e informal. Dentro de este panorama, Rinaudo (2014), destaca que las contribuciones más significativas desde la perspectiva socio-cultural del aprendizaje se desprenden de trabajos de Resnick (1987), Brown y colaboradores (1989), Bruner (1990, 1997), Cole (1999) y Lave y Wenger (1991), que en el campo de investigación de la psicología educacional fueron proporcionando conceptos $\mathrm{y}$ métodos de estudio relativos a los modos en que actúa la cultura sobre el desarrollo y el aprendizaje humano. Desde los enfoques socioculturales y su diversidad, se hace referencia al consenso que existe entre las distintas posiciones respecto de que las actividades humanas tienen lugar en contextos culturales, están mediatizadas por el lenguaje $y$ otras herramientas, consideran a los contextos cotidianos de aprendizaje y a las relaciones interpersonales; concibiendo también al aprendizaje como construcción de identidad (Rinaudo, 2014).

Mientras que la psicología ambiental es una disciplina que se preocupa por el abordaje de aquellas relaciones que se suceden entre los sujetos y el entorno natural, identificando aquellos procesos que surgen en relación a la persona con el ambiente que le rodea, lo cual implica considerar las percepciones, actitudes y representaciones en relación al ambiente (Martínez-Soto, 2019). En este sentido, Olivera Méndez (2015), añade que actualmente la agenda de la psicología ambiental considera diversas problemáticas como la migración, el cambio climático, la seguridad alimentaria y la escasez de los recursos, entre otros temas.

El propósito de este artículo no es definir exhaustivamente el campo de la 
psicología rural y sus interacciones con otras disciplinas y enfoques, sino visualizar que esta se erige como una disciplina que se encuentra en una tensión constante y a partir de una construcción social, situada e interdisciplinar en los ambientes.

Bruner (1997, 2003 en Rinaudo 2014) consideraba que la construcción de la identidad aparece ligada a la habilidad inherentemente humana de construir relatos, reclamando de forma reiterada que la escuela debía incorporar la modalidad narrativa en la enseñanza de las diferentes disciplinas, no sólo en las disciplinas literarias. En este sentido, Jackson (1995) plantea sobre la posibilidad de utilizar relatos en educación para lograr objetivos más profundos, que impliquen la construcción de valores, rasgos de personalidad y visiones del mundo, que se relacionen con lo que se quiere que los estudiantes sean como humanos.

Partir desde marcos culturales, invita a pensar a la ruralidad y educación desde un diálogo de saberes, una ecología, que como manifiesta de Sousa Santos (2010), está basada en el reconocimiento de la pluralidad de conocimientos heterogéneos - más allá del científico - y en las interconexiones continuas y dinámicas entre ellos. A su vez, el autor señala que todas las intervenciones en el mundo son valiosas, mencionando en este sentido, que la preservación de la biodiversidad estaría posibilitada por las formas y abundantes conocimientos rurales e indígenas; es decir, los modos de vida, los universos simbólicos y las sabidurías que se han preservado y sobrevivido a condiciones adversas, y que están basados completamente en la tradición oral.

Todas las formas de conocimiento mantienen prácticas y a su vez, constituyen sujetos; intervenciones alternativas posibilitadas por otras formas de conocimiento (de Souza Santos, 2010). En esta línea, Bruner (1986) postulaba sobre la posibilidad de construir mundos posibles a través de la experiencia y la imaginación; es a partir de estas ideas que pensamos que se puede atender y estudiar la coconstrucción y el conocimiento de nuestros mundos locales; por ello, en este escrito abordaremos las narrativas rurales de dos casos, dos personas que con sus saberes, prácticas y trayectorias son fundamentales para sus comunidades; considerando de esta manera a las narrativas como formas y sentidos para denotar aprendizajes como estrategias de enseñanza. Con el propósito de explorar y abordar las experiencias de aprendizaje a partir de la indagación y los puntos de vista de los actores locales en sus territorios y desde sus realidades socioproductivas. 


\section{Desarrollo}

En este apartado mencionaremos aquellos constructos que dotan de sentidos las expresividades que aquí desarrollaremos, temáticas tales como las comunidades de prácticas y las narrativas rurales; además se mencionarán los aspectos metodológicos del estudio y se expondrán algunos resultados preliminares.

\section{Marco teórico}

En este sentido encontramos estudios interesantes que hacen foco en las comunidades de prácticas y las historias de aprendizaje (Farnsworth, Kleanthous \& Wenger-Trayner, 2016; Garzón, 2020; Lave \& Wenger, 1991; Autora, 2019; Wenger, 2001; Wulandhari et al., 2021) y otros que refieren a la narratividad en educación (Barraza, 2014; Bruner, 1986; Leite Méndez, Rivas Flores y Cortés González, 2019; Otalvaro \& Velasquez, 2021; Reiss y McComas, 2020). Las comunidades de prácticas como espacios de co-construcción y narratividad compartida, y la consideración de los relatos rurales e interculturales en educación son aspectos teóricos que nos interesa resaltar en el marco de este estudio, centrándonos desde teorías del aprendizaje que amplían su visión y consideración al papel de la sociedad (Cherewka, 2019).

En cuanto a las comunidades de prácticas rurales, entre los estudios más recientes, se encuentra el trabajo de Wulandhani et al., (2021), que realizan un abordaje sobre el aprendizaje rural comunitario, arribando a discusiones que apuntan a la consideración de las comunidades para el desarrollo y la generación de innovaciones en los contextos rurales; logrando con su trabajo que los agricultores formen redes informales formadas por personas que comparten proximidad de dominios $y$ trayectorias.

Para ampliar en lo que respecta al concepto de comunidad de práctica, es necesario contextualizarlo, comentando que el mismo surgió a partir de un trabajo de Lave y Wenger (1991), en el que argumentaban principalmente que el aprendizaje no se basa en aspectos individuales, sino que es un proceso social que se sitúa en un contexto cultural e histórico. La teoría siguió construyendo corpus empírico con el estudio de una comunidad en una empresa de seguros (Wenger, 2001). Variados escritos concuerdan que una comunidad de práctica puede surgir en cualquier dominio, por ejemplo, en la práctica de crear nuevas formas de expresión artística, en las 
prácticas implicadas en la resolución de problemas climáticos, o las asociaciones de amigos de la escuela que están definiendo una identidad compartida; dándose de este modo, el aprendizaje a través de la participación en múltiples prácticas sociales (Farnsworth, Kleanthous \& Wenger-Trayner, 2016; Martín, 2019). De este modo, los diversos estudios de Wenger sobre el aprendizaje en contextos distintos al educativo formal han ayudado a pensar de manera diferente el aprendizaje en las escuelas (Farnsworth, Kleanthous \& Wenger-Trayner, 2016).

Los sistemas de aprendizaje social grupal se reflejan de manera útil en las comunidades de práctica, el término sugiere que el aprendizaje individual debe considerarse como emergente, lo que implica oportunidades para participar en las prácticas de la comunidad, así como en el desarrollo de una identidad que aporta sentido de pertenencia y compromiso. Las comunidades se consolidan a través de exposiciones de problemas grupales $\mathrm{y}$ creando un entendimiento común entre los miembros, con sus prácticas compartidas y vínculos personales que desarrollan con el tiempo. Uno de los aspectos es el mutuo compromiso, que se vincula a las relaciones y normas en torno a lo que los miembros hacen a través del proceso de interacción entre ellos. A su vez, al ser parte de la comunidad, las personas negocian significado, dando lugar a una responsabilidad mutua entre los participantes. En virtud de los repertorios compartidos que se desarrollan como prácticas comunes que los miembros adoptan a través de los procesos de interacción. Estas prácticas varían desde palabras, historias, acciones y formas de hacer las cosas hasta rituales y conceptos que la comunidad ha producido (Wulandhani et al., 2021).

Una de las dimensiones de las comunidades de práctica que plantea Wenger, refiere a la conservación y creación de conocimiento, considerando que se preservan las historias de aprendizaje como prácticas vivas, y se adaptan a las necesidades emergentes. La tensión inherente entre experiencias y competencias produce nuevos conocimientos (Martín, 2019).

A su vez, Wenger (2001), añade el concepto de corredores, definiéndolos como miembros que introducen nuevas prácticas dentro o entre comunidades, es por ello que, en esta investigación, identificamos a los corredores como aquellos narradores que desde su trabajo en las cooperativas rurales facilitan un cambio y fomentan nuevas perspectivas y prácticas innovadoras. En concordancia, y acorde a los planteos de construir conjuntamente 
una visión más modesta y cooperativa de la ciencia, se apunta al diálogo de saberes desde enfoques interdisciplinarios, con saberes locales e incluyendo a las comunidades afectadas (Svampa \& Viale, 2020).

Las interacciones sociales y las relaciones que se forjan con los compañeros de trabajo en el contexto de las cooperativas son las que permiten la consolidación, posibilitando la dinamización de los saberes; que reflejados en las comunidades de práctica posibilitan la construcción de historias compartidas y de vínculos interpersonales. En estos ambientes se van creando formas propias de comunicación, maneras de resolver los problemas que se presentan en lo personal y lo laboral, se genera así, un sentimiento de compromisos de equipo, que se afianza en el día a día consolidando una visión prospectiva alineada con la cooperativa (Garzón, 2020).

Las comunidades de prácticas como entidades dinámicas de aprendizajes, centradas en la participación social. Son unidades de análisis que permiten estudiar a las cooperativas como espacios de interacciones socio comunitarias que promueven la educación y el aprendizaje informal (Martín, Donolo \& Cugini, 2020).

Respecto a las narrativas en la ruralidad e interculturalidad interesa aquí hacer unas consideraciones generales de lo que una narración constituye, considerándola una de las formas más importantes de transmisión de saberes, a través de la cual se preserva la cultura y se transmiten aprendizajes a nuestros pares, siendo la narración el "puente" que permite que este proceso se materialice, por medio de la libre circulación de saberes (Bruner, 1997). Esto nos invita a pensarnos como narradores innatos, ya que, a través de la práctica, nos expresamos, sobrevivimos, sentimos $y$ permitimos que ciertos aprendizajes informales sigan "vivos" a través del tiempo y dejen una "huella" por medio de nuestras experiencias. El narrar, poder expresar o contar hechos, sucesos, anécdotas de las comunidades, es algo esencial para los seres humanos, ya que, de esta forma, las tradiciones, la cultura, las costumbres, las formas de vida, y los conocimientos aprendidos perduran en el tiempo (Barraza, 2014).

Las narrativas de los sujetos sobre situaciones, incidentes 0 recuerdos puntuales relacionados con la escuela vivida, nos acercan a otras formas de comprender el conocimiento, la experiencia, las relaciones, los contextos y la co-construcción de subjetividades. Trabajar con narrativas nos enfrenta con la complejidad del conocimiento y con la necesidad de avanzar en la comprensión 
del mismo. Una historia se construye en una trama a desentrañar, que articula componentes sociales, personales, políticos, culturales, ideológicos e históricos (Leite Méndez, Rivas Flores \& Cortes González, 2019).

Las narrativas son actos comunicativos que ponen en escena la intersubjetividad, la vida cotidiana, los roles sociales, identidades, compromiso mutuo, y el intercambio de intereses individuales y colectivos.

La fuerza de los relatos en educación es muy potente, así fue que, en 2003 en Brasil un conflicto en la escuela entre niños de zona urbana y de zona rural de São Carlos fue el precursor de una comunidad de aprendizaje. Existían muchas peleas, los estudiantes de la zona urbana trataban a los otros de una manera, menospreciándolos y bromeando, pero Rosa a través de la narración y sus relatos rurales logro que la brecha entre la ruralidad y la urbanidad pueda progresivamente subsanarse.

Cuando empezamos ya en 2003 con los grupos interactivos - que fue una fase de dos años - aparecen los sueños, las necesidades, planeamientos, cuando la comisión mixta se dedica a ver las prioridades. Vimos que los grupos interactivos eran la principal necesidad porque dos salas no tenían niños de zona rural, todas las otras sí. Entonces en todas empezamos con grupos interactivos en los que había por lo menos un familiar de zona rural, en este caso una mamá que se llamaba Rosa. Ella salía de un aula e iba para otra y luego para otra y en un mes estaba superado el conflicto. Fue muy impresionante porque el principio es humanizar las relaciones porque cuando hablamos del otro como otro inferior, no lo consideramos humano. Al tener ahí una madre que explica cosas, que cuenta cosas, los niños ya no se reían de las bromas, ya no hacían bromas... El efecto es muy efectivo y muy rápido. Antes los niños de la ciudad siempre decían que los otros eran mentirosos, que se inventaban historias... En una situación por ejemplo, ellos les decían: "No, mentira eso de que por la tarde cuando te vas a tu casa, te vas al río", no lo imaginaban. Rosa les decía: "Sí que van, van y se divierten ahí, y se bañan ahí". Y esto no era una fase, era un grupo interactivo y la madre iba explicando cosas de tal manera que cuando uno hacía una broma, otro le explicaba: "No, eso es verdad porque tal cosa, tal cosa y tal otra cosa". Y eso fue muy impresionante porque todo fue cambiado muy rápido. (Entrevista Rodrigues de Mello en Martín, 2016, p. 6)

En los usos y sentidos de incorporar las narrativas en educación, Reiss y McComas (2020), destacan la importancia de aprender informalmente sobre la naturaleza de la ciencia desde narraciones escritas y una visita a una propiedad histórica, lo que proporcionaría una imagen más completa de la vida y el trabajo de cada persona. Con esta idea de reunir dos fuentes de datos, los aprendices pueden apreciar cómo se construye la ciencia, aprender con la metodología compartida por todos los científicos, pero 
también incluyendo el conocimiento de las formas de trabajo idiosincrásicas en cada práctica.

\section{Aspectos metodológicos}

La investigación cualitativa pretende acercarse al mundo de "ahí fuera", y entender, describir y explicar fenómenos sociales "desde el interior" en variadas formas; una de esas refiere al análisis de las distintas experiencias de las personas o sus comunidades, experiencias que se pueden relacionar con historias de vida biográficas o con prácticas cotidianas, analizando de esta forma, el conocimiento cotidiano, los saberes e historias (Flick, 2007).

Con la intención de comprender desde las narrativas las formas y sentidos que denotan los contextos rurales, se realizó un estudio de caso múltiple (Stake, 1998). El estudio de caso cualitativo valora las múltiples perspectivas de los interesados, la observación en circunstancias que se producen de forma natural y la interpretación en cada contexto (Simons, 2011).

En el presente trabajo, se consideraron dos casos de personas que son parte de cooperativas situadas en contextos rurales; las narrativas sobre las prácticas rurales buscan describir experiencias personales, desde el ámbito sentimental, educativo y comunitario.

Participantes: Por un lado, una de las narrativas es de la secretaria de una cooperativa trabajo artístico $\mathrm{y}$ permacultural que tiene su sede eco productiva en la zona del cerro Ñu Porá en Río Ceballos, provincia de Córdoba, Argentina. La organización desde 2013 desarrolla sus actividades respetando las características del ecosistema natural, la agricultura y la cultura permanente; es única en su tipo en Argentina y está compuesta por aproximadamente ocho asociados. Se dedica a elaborar una variedad de producciones y realiza tres tipos de servicios: artísticos, con animaciones para fiestas a través de la murga o la pintura; bio-construcción, con la construcción de huertas permaculturales, asesoramiento de saneamientos ecológicos, talleres variados y abiertos al público sobre compostaje, biopiscinas y baños secos; y elaboración de productos para ferias, con premezclas multirrecetas para tortas $\mathrm{y}$ sopas, combinación de semillas, semillas para huertas agroecológicas, bolsos reutilizables, ecobancos, entre otros productos.

Otra de las narrativas es la del presidente de una cooperativa agrícola tambera que se ubica en la localidad de James Craik en el departamento Tercero 
Arriba, también de la Provincia de Córdoba, Argentina. La cooperativa de la que forma parte surgió en 1926 y se encuentra inmersa en la llanura pampeana. El número de socios activos de la cooperativa es de aproximadamente 35, figurando un centenar en el padrón. El principal producto que se elabora es el queso, pero también se cuenta con una planta de secado con la que se brinda servicio y subproductos - suero, suero entero en polvo y desmineralizado.

Herramienta de recolección de datos: Las narrativas se configuran en estos contextos como una opción metodológica e investigativa que permite revelar lo que existe en los testimonios subjetivos de las personas, que están cargados de eventos que son valorados e interpretados, dando sentido a sus vivencias $y$ a sus aprendizajes. A su vez, se considera que las entrevistas narrativas son una forma artesanal de comunicar experiencias subjetivas, su carácter desestructurado es una herramienta que permite comprender las experiencias y los contextos en que fueron co-construidas, así como aquellos aspectos que producen los cambios y motivan las acciones (Arias Guevara, 2020).

Para la comprensión de los aspectos personales y atendiendo a las trayectorias, se realizaron entrevistas que se centraron en la historia de vida, solicitando a cada persona el relato de las experiencias destacadas y los significados que le atribuyó a esas situaciones (Sautu, 1999; Taylor \& Bogdan, 1992), averiguando sobre lo que creen, hacen, que sienten cuando lo hacen y los significados que le atribuyen a sus prácticas rurales; describiendo experiencias personales desde el ámbito sentimental de las relaciones e interdependencias.

Procedimientos: Luego de un primer contacto y de charlas informales, las entrevistas en profundidad se llevaron a cabo en los contextos de trabajo, en las cooperativas de cada socio.

\section{Resultados}

Los resultados de este estudio apuntan a pensar en tres dimensiones: trayectorias de participación en territorio, educación para y con la comunidad y prácticas cooperativas y soberanas. Estos aspectos permiten analizar las narrativas y avanzar hacia una interacción en diversas ecologías y contextos.

\section{Trayectorias de participación en territorio}

En cuanto a las trayectorias participativas de cada persona en su entorno rural, resulta interesante conocer el devenir de prácticas posibilitadoras de otras formas de conocimiento. Asumiendo 
las proporciones de una perspectiva sobre la particularidad relacional del conocimiento y el aprendizaje, sobre el carácter negociado del significado y acerca de la naturaleza comprometida orientada por problemas de la actividad de aprendizaje (Lave \& Wenger, 1991); en este contexto, no hay actividad que no esté situada, la trayectoria de estos cooperativistas transcurre en su participación continua y cotidiana en los contextos rurales de los que forman parte.

Las narrativas que apuntan a las trayectorias de participación laboral dentro de cada cooperativa responden en conjunto a diversas vivencias que se entrelazan $y$ resignifican de múltiples formas, con una fuerte impronta emocional dan sentido y permiten desarrollar nuevos modos de mirar, sentir, comprometerse y situarse en el mundo.

A continuación, se comparten dos fragmentos de las autoridades de las cooperativas a modo de poder entender su inclusión y participación.

...yo trabaje en el campo, mi viejo tenia tambo, vengo de toda esa rama y el hecho de empezar a trabajar en la cooperativa me ha abierto la cabeza mil por ciento, me ha contactado con mucha gente. Realmente uno dimensiona el conocimiento que hay hacia la cooperativa, uno de afuera " $y$ es una cooperativa más de acá" en cambio uno viviéndolo acá dentro dice "no, esto tiene una identidad, tiene una fuerza, tiene un compromiso, tiene interés"
(Fragmento de la narrativa del Presidente Cooperativa Agrícola Tambera).

Y básicamente un hecho que fue bisagra... tuvimos un episodio en una casa en Córdoba, una casa quinta que estaba en la ciudad que colindaba con $\mathrm{C}$-la marca de una gaseosa- y esa quinta se vendía y cuando se vende nos dicen: "chicos se tienen que ir porque acá vamos a vender", entonces nos quedamos mientras se llevaban todos los materiales de la casa que estaban desarmando, porque nosotros nos enfocábamos solo en lo artístico ... Pero cuando un día nos despertamos y vimos que estaban bajando todos los árboles y que esto que el hombre nos estaba diciendo era verdad, icah era verdad que iban a voltear la casa?! Ah bueno, bien, fue muy duro, al punto de llorar en la cama mientras tiraban los árboles. Y ver toda esa realidad y comenzar a pensar que si estos árboles los vende el viejito de al lado, que era nuestro ídolo del campo que estaba re chocho porque vendía los árboles, ¿a quién se los vendía? a los cortaderos de ladrillo, ah bien...y si estos árboles van al cortadero de ladrillo, ¿que implica esto? (se toca la ropa) ¿y esto? ¿Y eso? ¿Y aquello? Y entonces empezamos a ver que todo lo que teníamos implicaba destruir algo en algún lugar del planeta, y eso fue los que nos llevó a pensar más desde el reciclaje. Desde el principio pensando que el reciclaje era lo mejor del mundo, porque hasta lo que podíamos ver en ese momento era lo mejor del mundo, quizá para el aquí y ahora nuestro de ese momento lo fue, pero hoy para nosotros el reciclaje es la última opción. Fue una cadena que se fue dando sin querer queriendo y nunca nos imaginamos que íbamos a terminar haciendo todo esto, o quizá en algún punto sí porque lo elegimos ... Ahora estamos en este momento de empezar a integrar todo esto. Si tuviera que decir en síntesis porque vinimos a 
laburar de todo esto es por un quiebre fuerte que te hace cambiar el chip y dar vuelta la tortilla (Fragmento de la narrativa de la Secretaria de la Cooperativa de Arte y Permacultura).

En el caso del presidente, fue construyendo sus trayectos desde la ruralidad y la integración a la cooperativa desde joven, el trabajo en el tambo con su padre y la participación en territorio. En cambio, la secretaria de la otra cooperativa, comenta que frente a las adversidades $\mathrm{y}$ situaciones que denotaban destrucción del ambiente, fueron otorgándole otros sentidos a sus prácticas desde la cooperativa, considerando el reciclaje primero, y la permacultura luego como forma de vida asociativa.

En los fragmentos seleccionados se manifiestan decisiones, sentires y cotidianidades laborales en el contexto de cada cooperativa, recuperándose lo invisible, valioso y genuino; $\mathrm{y}$ aquellos lugares, eventos y comunidades completas que dieron $\mathrm{y}$ dan una oportunidad a la búsqueda, la transformación y la reconstrucción de procesos emocionales y sociales, exponiendo impronta y la intimidad de la experiencia ofrecida en el entorno (Alliaud \& Suarez, 2011 en Otalvaro \& Velasquez, 2021).

Podemos decir, que los participantes de estas cooperativas construyen su identidad y territorialidades a partir de sus prácticas cotidianas y su participación en contexto.

\section{Educación para y con la comunidad}

Un aspecto central de estas narrativas informales es su alcance y su importancia educativa. En este punto por un lado, puede analizarse al interior de la cooperativa aquellos propósitos educativos que las consolidan, y por otro lado, como las cooperativas generan y trascienden en la sociedad con la creación de espacios y oportunidades de aprendizajes.

Es preciso manifestar que en ambos casos las asambleas son espacios de participación democrático, inclusivo y crítico, que permiten a los socios y a la sociedad en general preguntar, co-construir su identidad cooperativa, generar vínculos y responsabilidad social.

En los fragmentos que continúan los socios mencionan como las cooperativas son espacios de aprendizaje y educación, y se co-construyen a partir de formas de entender a la cooperación.

Y la coope surge de laburar tantos años como CC, porque nosotros antes nos llamábamos CC servicios artísticos. Después de haber laburado años, años, años, que mucha gente nos decía che por qué no se arman como una asociación civil, una fundación, etc... y nosotros decíamos no porque eso tarda mucho, y un día nos dimos cuenta que veníamos laburando hacia 15 años y dijimos che, ¿y si lo hacemos? ... Empezamos 
a pensar qué formato nos gustaría y ahí vino toda la historia de fundación, asociación civil ... fue el punto de hincapié que nos hizo pensar en una cooperativa, también laburamos mucho la educación popular y empezamos a verlo mucho más holística...cerraba con nuestros ideales mucho más lo que es la lógica del cooperativismo. $\mathrm{Y}$ por último cuando entramos en todo lo de la permacultura dijimos ¡wow! no podríamos haber elegido mejor formato que la cooperativa porque realmente es permacultural...o por lo menos lo puede ser (Fragmento de la narrativa de la Secretaria de la Cooperativa de Arte y Permacultura).

Tenemos un área de recursos humanos pero el área de recursos humanos es puerta para dentro, se encarga de capacitar a los empleados nuestros, se encarga del funcionamiento en sí pero no abarca al socio por ejemplo, nadie de recursos humanos del departamento selecciona personal. (Fragmento de la narrativa del Presidente Cooperativa Agrícola Tambera).

Si bien los fragmentos son escuetos y no desentrañan todos los aspectos educativos de estas cooperativas, es importante resaltar que el socio de la cooperativa agrícola tambera manifiesta que no existe una comisión educativa en la organización pero que sería muy promisorio e importante tener una. La mayoría de la capacitación se genera desde el área de recursos humanos intra cooperativamente. En tanto, desde el caso de la cooperativa de arte y permacultura, la perspectiva de la educación popular se encuentra en concordancia con los principios y valores de una construcción democrática cooperativa permacultural.

En cuanto a lo educativo que las cooperativas desarrollan y extienden a la población, desde la cooperativa de arte y permacultura se fomenta la construcción de un espacio de escuela monte con sede en el Cerro de Ñu Porá de Río Ceballos; con la participación y generación de diversos encuentros (ferias, talleres, y charlas), $\mathrm{y}$ con la posibilidad de generar contenidos.

El siguiente segmento muestra una de las acciones desarrolladas por la cooperativa, en la que se hibrida arte, educación y permacultura.

\begin{abstract}
Veníamos con una obra de teatro que se llamaba Tierra Viva, una obra que empezó siendo una muestra para escuelas de danza y música. Queríamos contar una leyenda, no encontramos una hermosa leyenda que nos representara realmente porque, o era dios o era el diablo, las leyendas eran un montón y todo malo o bueno (Fragmento de la narrativa de la Secretaria de la Cooperativa de Arte y Permacultura).
\end{abstract}

Respecto a la cooperativa agrícola ganadera, el propósito central está puesto en generar acciones para que participen nuevos miembros; el presidente comenta que han ido estudiantes de educación y la universidad a realizarle entrevistas 
revalorizando de esta forma la proyección de las acciones.

Este año a través del Ministerio de Agricultura de la Provincia ... generó unas capacitaciones para jóvenes cooperativistas que era enseñarles cuáles eran los puestos, como trabaja una cooperativa, fue muy importante. Yo considero que no tiene... si bien justamente si uno viene de una cooperativa puede dar la enseñanza de lo que sabe ... es necesario porque es algo que nos está pasando -como te decía- ahora a nosotros. Nosotros venimos renovando una, dos personas por año por consejo, es muy difícil, hay muy poco interés. Buscamos gente joven, buscamos hijos de productores y bueno este año por ejemplo, creo que vamos a conseguir dos que nos renueven, tenemos gente grande en el consejo que ya nos ha dicho "che, si hay alguien que me remplace yo quiero que entre gente nueva" y nosotros decimos "sí nosotros también pero nos cuesta". (Fragmento de la narrativa del Presidente Cooperativa Agrícola Tambera).

\section{Prácticas cooperativas y soberanas}

En lo que respecta a prácticas cooperativas y soberanas que se proyectan más allá de la comunidad implicada, dos fragmentos de las narrativas de los socios permiten vislumbrar como una construcción social y cultural debe hacerse más allá de los actores involucrados; implicando un compromiso social y metas expandidas.

Eso nace a través de la FECOFE en reuniones que hemos tenido de consejo que coincide justo cuando se vota la ley de emergencia alimentaria, coincidimos un grupo de cooperativas que teníamos que dar el ejemplo. En realidad esto más allá de la contribución social que nosotros podemos hacer, que es mínima, cubrimos una partecita muy chiquita del déficit alimentario que hay pero nosotros lo tuvimos pensado como un disparador a generar en otras empresas privadas, no solo todas tienen que ser cooperativas, no todo tiene que ser empresas públicas sino también empresas privadas y decir "bueno mirá de mi producción yo destino el uno por ciento, el dos por ciento, el tres, no sé, lo que pueda a esta contribución social" que es básicamente poner el producto al mejor precio posible disponible ... nosotros dimos un primer paso de poner productos sobre todo en Buenos Aires a un precio accesible pero en realidad lo que nosotros buscamos era hacer un disparador, generar un movimiento, que si se suma el resto de las empresas con una pequeña partecita no tendríamos ningún problema, no haría falta la ley de emergencia alimentaria. Se cubriría tranquilamente, si no cumple su objetivo simplemente que se vayan sumando con el paso del tiempo creo que se va a lograr ... la UTT -Unión de Trabajadores de la Tierra- nos pareció un medio muy importante ... La semana pasada estuvo el jefe comercial nuestro en Buenos Aires, entonces ellos nos pidieron que si teníamos un momentito que ellos querían capacitar a sus vendedores con respecto a la elaboración de quesos, cómo se hacían, qué diferencias había entre uno y otro para salir a ofrecer esos productos. Esa unión de trabajadores está muy comprometida, muy interesados y muy agradecidos también porque ellos participaron de la firma de convenio y la verdad que en poquito tiempo pudimos empezaron a llevar productos, así que esperemos que tenga buenos resultados, esperemos que se sume gente que eso es lo importante 
(Fragmento de la narrativa del Presidente Cooperativa Agrícola Tambera).

Cuando entendes que la pacha es tu casa, ya no tenés que tener tu patio para sembrar, donde estas vas a sembrar o vas a generar cosas en pos de eso, en relación a eso. A las plantas, a cualquier lugar del mundo. Eso es recordar, todos lo tenemos, no es que me pasó a mí, hay que abrirse para recordar. El alimento hace mucho, todo este cambio no lo hubiéramos hecho si no comiéramos por lo menos en un 80 por ciento sin agrotóxicos, participamos de compras comunitarias cada seis meses de todos productos orgánicos. La pre mezcla surgió como un agradecimiento de todo esto, miramos y teníamos todos tachos de porotos, garbanzos, etc. y vimos todo orgánico y dijimos somos muy privilegiados y dijimos para que haya más privilegiados tenemos que agradecer esto. Pero no agradecer de decir gracias, gracias, sino hacer algo (Fragmento de la narrativa de la Secretaria de la Cooperativa de Arte y Permacultura).

Como se exponen en los dos fragmentos, la importancia radica en construir nuevas respuestas colectivas en las comunidades, tomando la iniciativa y decisiones; como manifiesta el Movimiento Campesino Internacional (2003) y Martínez (2010), hay que otorgarle un valor prioritario a las producciones locales de alimento para la sociedad y las comunidades tienen el derecho de proteger, apoyar y promover sus propios sistemas de producción agraria.

En concordancia con lo planteado
Martín (2020), podemos decir que los narradores sitúan su aprendizaje no sólo como un proceso participativo y colectivo a una escala comunitaria centrada en sus cooperativas, sino también como aprendizajes fundados en valores que se corresponden con un marco sociocultural; estimulando la co-construcción de saberes trascendentales que tengan un impacto en el ambiente. A partir de todas las entrevistas se vislumbra la importancia y vinculación aquellas prácticas llevadas a cabo en las cooperativas con el concepto de soberanía alimentaria, en términos de una construcción soberana y autónoma con formas de producción, consumo y distribución de los alimentos -una necesidad básica y constitutiva de la existencia- en función de modelos productivos a gran escala.

\section{Consideraciones finales}

¿Qué queremos expresar con estas ideas? Por una parte, queremos manifestar sobre la relevancia que tiene en educación considerar las narrativas o relatos autobiográficos para el aprendizaje rural e intercultural; desde la escuela, conocer las posibilidades y las prácticas de los actores locales posibilitaría pensar y tomar conocimiento sobre lo que ocurre en las cooperativas próximas, o bien conocer que

por Perroni Gasull, Sago Herrador y 
prácticas permaculturales y sustentables se están generando a la vuelta de la esquina.

Por otra parte, pensamos que conocer estos relatos locales $\mathrm{y}$ en territorio permitirían a las comunidades co-construir procesos de conocimiento; escuchar los comienzos del presidente de la cooperativa de agrícola tambera, una asociación de aproximadamente 100 años, como conocer los motivos que llevaron a los socios de la cooperativa de permacultura a su creación, son hitos relevantes para las historias de los participantes y las comunidades cooperativas-.

Las trayectorias personales $\mathrm{y}$ grupales que se co-construyen en el territorio, el relieve en los aspectos educativos y la consideración de prácticas más cooperativas y soberanas son dimensiones importantes para analizar en el marco de la generación de ambientes educativos promisorios. Entre los resultados de este estudio, se analizó que las trayectorias de participación dentro de cada cooperativa responden en conjunto a diversas vivencias que se entrelazan y resignifican de múltiples formas. Además de los contextos formales de aprendizaje, existen otros espacios como las cooperativas, en las que transcurren eventos y situaciones en las que las personas participan. En el interior de cada cooperativa se perfilan propósitos de aprendizaje y educativos que permiten su consolidación, de igual modo que las cooperativas generan y trascienden en la sociedad con la creación de otras situaciones u oportunidades de aprendizajes.

En lo que respecta a prácticas cooperativas más soberanas, la construcción social y cultural se desarrolla más allá de los actores involucrados y el territorio, trascendiendo a las comunidades con impacto social.

En la medida que cada cooperativa crece y se desarrolla en el tiempo, se construyen historias compartidas, sobre los comienzos, las dificultades y las oportunidades futuras; desenvolviéndose una identidad narrativa, en lo personal y colectivo; convirtiéndose aquellas historias de participación personal en parte de las historias e identidad de cada comunidad (Wenger, Trayner \& de Laat, 2011).

Las teorías del aprendizaje están basadas en concepciones fundamentales acerca de la persona, el mundo y sus relaciones; el aprendizaje es una dimensión de la práctica social (Lave \& Wenger, 1991). El aprendizaje informal puede ayudar a los estudiantes a apreciar aspectos importantes y actuar como un puente sólido para vincular el mundo relativamente breve de la escuela con el 
aprendizaje permanente (Reiss \& Mccomas, 2020).

En consonancia, las estrategias pedagógicas de los educadores deberían reflejar metodologías orientadas al diálogo y dejar espacio para que los estudiantes examinen sus propias experiencias (Cherewka, 2019).

¿Por dónde seguir? En este contexto, sería relevante pensar en términos de novedosas prácticas disruptivas realizadas en entornos no convencionales. A su vez, en psicología rural, así como en psicología educativa, social y ambiental, se requiere estudiar, analizar y comprender más el ambiente rural, ya que la complejidad de la problemática ambiental rural demanda un trabajo interdisciplinario (Olivera Méndez, 2015).

Entre los principales aportes, con el estudio de las narrativas rurales en contextos no convencionales como las cooperativas, se habilitan otras formas $\mathrm{y}$ abordajes respecto a los tradicionales. El desafío en esta área se encuentra en pensar otras formas de hacer investigación educativa rural, por un lado, desde la diversidad del contexto, cuando se piensa en situaciones de aprendizaje, por lo general, se piensa en las escuelas rurales y no en las cooperativos como contextos informales de aprendizaje. Por otro lado, las teorías del aprendizaje contemporáneas están habilitando otras formas de investigar sobre el aprendizaje a través de metodologías más vividas y experienciales, dando el puntapié para el desarrollo de teorías más sólidas del aprendizaje elaboradas desde lo biográfico y situado (Alheit, 2018).

Para finalizar, compartimos un relato del diario de Oliver Sacks (2017), en el que relata sobre un viaje de un grupo de botánicos, profesionales y aficionados unidos por la pasión y búsqueda de helechos en Oaxaca, México; lo que nos anima a seguir construyendo y narrando relatos de los aprendizajes informales de nuestras vidas.

La tarea, o el capricho, que me he impuesto de llevar un diario está llegando a su fin. Me asombra que lo haya mantenido con tanta pertinancia, pero ésta es mi pasión: verter la realidad en palabras. He tomado estas últimas notas sentado, no bajo un ahuehuete sino bajo un nopal, y John Bristow ( $\mathrm{e}$ el tercer John del grupo!, tan obsesivo con la cámara como lo soy yo con la pluma) me ha hecho en silencio una foto cuando creía que no le miraba. Los largos rayos del sol poniente doran los pueblecitos zapotecas y las iglesias del siglo XVI, una tierra agradable, plácida, suavemente ondulada. La excursión ha sido encantadora. No me lo había pasado tan bien durante una de estas salidas desde hacía muchos años, pero tampoco puedo analizar en estos momentos, a qué se debe tanta...tanta perfección (Sacks, 2017, p. 174). 


\section{Referencias}

Alheit, P. (2018). Biographical learning within the lifelong learning discourse. En Illeris, K. (Ed.). Contemporary Theories of Learning Theorists ... In Their Own Words (pp. 153-165). Taylor y Francys Group. https://doi.org/10.4324/9781315147277-11

Arias Guevara, M. (2020). Otra mirada para la otra economía: cuando el lente nos llega desde los otros feminismos. Revista Estudios del Desarrollo Social: Cuba y América Latina, 8(3), 14. Recuperado de: http://www.revflacso.uh.cu/index.php/EDS /article/view/505

Barraza, J. (2014). Contar según Jerome Bruner. Itinerario Educativo, XXVIII, (63), 31-59. https://doi.org/10.21500/01212753.1480

Barron, B., \& Bell, P. (2016). Learning environments in and out of school. En Corno, L., \& Anderman, E. (Eds.). Handbook of Educational Psychology. Tercera Edición (pp. 323-335). Nueva York: Routledge.

Bruner, J. (1997). La educación puerta de la cultura. Madrid: Visor Dis. S.A.

Bruner, J. (1986). Realidad mental y mundos posibles: los actos de la imaginación que dan sentido a la experiencia. Barcelona, España: Gedisa Editorial.

Cherewka, A. (2019). What's in a Learning Theory? A Comparison of Contemporary Learning Theories, Their Shared Elements, and Their Vision of Society's Role in Learning, En Adult Education Research Conference, Búfalo, New York. Recuperado de: https://newprairiepress.org/aerc/2019/paper $\underline{\mathrm{s} / 12}$
De Sousa Santos, B. (2010). Descolonizar el saber, reinventar el poder. Montevideo: Editorial Trilce.

Farnsworth, V., Kleanthous, I., \& WengerTrayner, E. (2016). Communities of Practice as a Social Theory of Learning: a Conversation with Etienne Wenger. British Journal of Educational Studies, 64(2), 139160.

https://doi.org/10.1080/00071005.2015.11 $\underline{33799}$

Flick, U. (2015). El diseño de investigación cualitativa. Madrid: Morata.

Garzón, M. (2020). Las comunidades de práctica en las organizaciones. Desarrollo gerencial, $12(1), \quad 1-22$. https://doi.org/10.17081/dege...3683

Jackson, P. (1995). Sobre el lugar de la narrativa en la enseñanza. En McEwan, H., \& Egan, K. (Eds.). La narrativa en la enseñanza, el aprendizaje y la investigación (pp.25-51). Buenos Aires: Amorrortu editores.

Landini, F. (2015). La noción de psicología rural y sus desafíos en el contexto latinoamericano. En Landini, F. et al. (Eds.). Hacia una psicología rural latinoamericana (pp. 21-32). Ciudad Autónoma de Buenos Aires: CLACSO.

Lave, J., \& Wenger, E. (1991). Situated Learning: $\quad$ Legitimate Peripheral Participation. Cambridge University Press: CambridgeUK. https://doi.org/10.1017/CBO97805118153 $\underline{55}$

Leite Méndez, A., Rivas Flores, I., \& Cortés González, P. (2019). Narrativas, enseñanza y universidad. En Martín, R.B., Rinaudo, M. C., \& Paoloni, P. V. (Eds.). Comunidades. Estudios y experiencias sobre contextos y comunidades de aprendizaje (pp. 63-73). Villa María, Argentina: EDUVIM. 
Martín, R. (2019) Perspectiva teórica sobre el estudio de los contextos y comunidades de aprendizaje. En Martín, R., Paoloni, P. V., \& Rinaudo, M. C. (Comps.) Comunidades. Estudios y experiencias sobre contextos y comunidades de aprendizaje (pp. 75-132). Eduvim.

Martín, R. (2016). Expresiones sobre la creación de comunidades de aprendizaje transformadoras en Brasil. Relato de la especialista Roseli Rodrigues de Mello. Revista Educación, Formación e Investigación, 2(4). Recuperado de: https://ppct.caicyt.gov.ar/index.php/efi/arti cle/view/9722/9032\#

Martín, R. B., Donolo, D., \& Cugini, A. (2020). Community of practice and green learning. The case of a Permaculture Art Cooperative. COODES. Cooperativismo y Desarrollo, 8(2), 263-281. http://coodes.upr.edu.cu/index.php/coodes/ article/view/320

Martínez, R. (2010). Soberanía agroalimentaria: características, obstáculos y perspectivas. Ciencia y Sociedad, 35(4), 623-656.

https://doi.org/10.22206/cys.2010.v35i4.pp $\underline{623-656}$

Martínez-Soto, J. (2019). La ciudad: una visión desde la psicología ambiental. Quivera. Revista de Estudios Territoriales, 21(1), 43-57. https://doi.org/10.36677/qret.v21i1.11490

Olivera Méndez, A. (2015). Psicología ambiental y ruralidad. En Landini, F. et al. (Eds.). Hacia una psicología rural latinoamericana (pp. 307-314). Ciudad Autónoma de Buenos Aires: CLACSO.

Otálvaro, O. P. R., \& Velásquez, F. A. A. (2021). Experiencias profesionales en la ruralidad. Revista Argentina de Investigación Narrativa, 1(1), 222-236.
Perroni Gasull, C., Sago Herrador, E., \& Belén Martín, R. (2020). Aprendizagem informal e práticas agroecológicas em o contexto de um pomar. Revista De Ensino De Biologia Da SBEnBio, 13(1), 206-222. https://doi.org/10.46667/renbio.v13i1.344

Reiss, M. J., \& McComas, W. F. (2020). Informal Learning Sites and Their Role in Communicating the Nature of Science. En McComas, W. (Ed.). Nature of Science in Science Instruction (pp. 711729). Cham: Springer. https://doi.org/10.1007/978-3-030-572396_39

Rinaudo, M. C. (2014). Estudios sobre los contextos de aprendizaje. Arenas y fronteras. En Paoloni, P., Rinaudo, M., \& González Fernández, A. (Eds.). Cuestiones en Psicología Educacional: cuestiones teóricas, metodológicas y estudios de campo (pp.163-205). Tenerife: Sociedad Latinoamericana de Comunicación Social (SLCS).

Sacks, O. (2017). Diario de Oaxaca. Barcelona, España: Anagrama.

Sautu, R. (1999). El método biográfico. La reconstrucción de la sociedad a partir del testimonio de los actores. Buenos Aires: Editorial de Belgrano.

Simons, H. (2011). El estudio de caso: Teoría y práctica. Madrid: Morata.

Stake, J. (1998). Investigación con estudios de caso. Madrid: Morata.

Svampa, M., \& Viale, E. (2020). El colapso ecológico ya llegó. Buenos Aires: Siglo XXI Editores.

Taylor, S., \& Bogdan, R. (1992). Introducción a los métodos cualitativos en investigación. La búsqueda de los significados. España: Editorial Paidós. 
Via Campesina. Movimiento Campesino Internacional. Recuperado de: https://viacampesina.org/es/quignificasoberanalimentaria/

Wenger, E. (2001). Comunidades de práctica. Aprendizaje, significado $e$ identidad. Barcelona: Paidos Ibérica, S. A.

Wenger, E., Trayner, B., \& De Laat, M. (2011). Promoting and assessing value creation in communities and networks: A conceptual framework. The Netherlands: Ruud de Moor Centrum.

Wulandhari, N. B. I., Mishra, N., Dora, M., \& Samuel, F. W. (2021). Understanding rural Do-It-Yourself science through social learning in communities of practice. Technological Forecasting and Social Change, 163. https://doi.org/10.1016/j.techfore.2020.120 $\underline{411}$

Información del articulo / Article Information

Recibido en: 30/03/2021

Aprobado en: 04/05/2021

Publicado en: 12/07/2021

Received on March 30th, 2021

Accepted on May 04th, 2021

Published on July, 12th, 2021

Contribuciones en el artículo: Los autores fueron responsables de todas las etapas y resultados de la investigación, a saber: elaboración, análisis e interpretación de los datos; redacción y revisión del contenido del manuscrito y; aprobación de la versión final publicada.

Author Contributions: The author were responsible for the designing, delineating, analyzing and interpreting the data, production of the manuscript, critical revision of the content and approval of the final version published.

Conflictos de Intereses: Los autores han declarado que no existe conflicto de intereses con respecto a este artículo.

Conflict of Interest: None reported.

Evaluación del artículo

Artículo evaluado por los revisores.

\section{Article Peer Review}

Double review

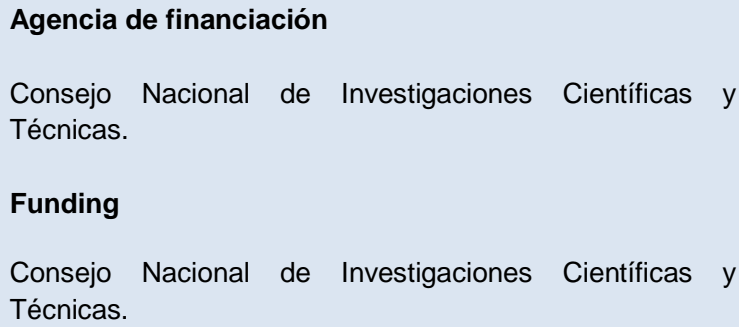

Cómo citar este artículo / How to cite this article

APA

Martín, R. B., Foresto, E., \& Donolo, D. S. (2021).

Comunidades y aprendizaje informal: Las narrativas rurales de dos cooperativistas. Rev. Bras. Educ. Camp., 6, e11885. http://dx.doi.org/10.20873/uft.rbec.e11885

ABNT

MARTÍN, R. B.; FORESTO, E.; DONOLO, D. S. Comunidades y aprendizaje informal: Las narrativas rurales de dos cooperativistas. Rev. Bras. Educ. Camp., Tocantinópolis, v. 6, e11885, 2021. http://dx.doi.org/10.20873/uft.rbec.e11885 